\title{
Examples of global attractors in parabolic problems
}

\author{
Alexandre N. Carvalho*, Jan. W. Cholewa and Tomasz Dlotko
}

(Received February 26, 1996; Revised July 2, 1996)

\begin{abstract}
Based on the theory of dissipative systems (see [HA]), necessary and sufficient conditions for the existence of global attractors for semilinear parabolic problems are studied. Many examples are considered to show precisely how this conditions works. We deal in particular with the Hodgkin-Huxley, Fitzhugh-Nagumo and Lotka-Volterra systems of reaction-diffusion equations, 2-D Navier-Stokes and Burgers equations of hydrodynamics and the Cahn-Hilliard pattern formation equation.
\end{abstract}

Key words: parabolic systems, global solutions, dissipative semigroups, global attractors.

\section{Introduction}

In this paper we consider an initial boundary value problem for an autonomous semilinear parabolic system of the form:

$$
\left\{\begin{array}{l}
u_{t}=-\mathcal{A} u+f\left(x, u, D^{1} u, \ldots, D^{k} u\right), t>0, x \in \Omega \subset R^{n}, \\
\mathcal{B}_{j} u=0, t>0, x \in \partial \Omega, j=1, \ldots, m d, \\
u(0, x)=u_{0}(x), x \in \Omega .
\end{array}\right.
$$

Both $\mathcal{A}:=(-1)^{m} \sum_{|\alpha| \leq 2 m}\left[a_{\alpha}^{r s}(x)\right]_{d \times d} D^{\alpha}$ and $\mathcal{B}_{j}:=\sum_{|\beta| \leq m_{j}}\left[b_{\beta}^{r s}(x)\right]_{1 \times d} D^{\beta}$ $(j=1, \ldots, m d)$ are linear matrix differential operators acting on $u=$ $\left(u_{1}, \ldots, u_{d}\right): R^{+} \times \Omega \rightarrow R^{d}, \Omega$ is a bounded domain in $R^{n}$ with a regular boundary $\partial \Omega \in C^{2 m}, k \leq 2 m-1$ is a fixed nonnegative integer and $D^{j}(j=1, \ldots, k)$ denotes a vector of partial derivatives with respect to $x$ of order $j$, i.e.

$$
D^{j} u=\left\{D^{\sigma} u_{1}, \ldots, D^{\sigma} u_{d}\right\}_{|\sigma|=j} .
$$

We assume that:

A-I The triple $\left(\mathcal{A},\left\{\mathcal{B}_{j}\right\}, \Omega\right)$ forms a regular parabolic initial boundary value problem of $2 m$-th order in the sense of [AM, Chapt. 1, Sec. 1].

1991 Mathematics Subject Classification : 35B40, 35K50, 35G30, 35Q30, 35Q35.

${ }^{*}$ Partially supported by CNPq - Brazil, under the grant \#300.889/92-5. 
A-II All components of the nonlinear term $f=\left(f_{1}, \ldots, f_{d}\right)$ are real valued functions, locally Lipschitz continuous with respect to each of its arguments separately.

Problems of type (1) appear frequently in applications and hence the existence of solutions as well as their behavior in time are an important factor in theoretical considerations. For sufficiently large $p$ conditions AI, A-II guarantee existence of local $\left[L^{p}(\Omega)\right]^{d}$-solutions of (1) in fractional power spaces $X^{\alpha}=D\left(A^{\alpha}\right)$ with $\alpha \in[0,1)$ close to one. Whenever the problem (1) generates a global semiflow $\{T(t)\}$ on $X^{\alpha}$, it is the study of the global attractor [HA] for $\{T(t)\}$, which allows then to reveal much of the relevant information about the system. In the case of semigroups created by abstract evolutionary equations, the necessary and sufficient condition for the existence of a global attractor has been formulated in [CD3], where some basic applications to parabolic p.d.e.'s have also been mentioned ([CD3, Rem. 1]). However, since the latter has been treated there merely in a short cut, it is the aim of the present paper to show precisely how the conditions of [CD3] work in applications.

Our special concern here is the case when (1) produces a semiflow which is not dissipative [HA] on the whole phase space but only on a subset of it. This may happen, for example, when (1) generates global semiflow of globally bounded solutions but with an unbounded set of equilibria (as for the Cahn-Hilliard equation). Nevertheless it is also possible the situation when some initial conditions produce solutions which 'blow-up' in finite or infinite time, whereas for other initial conditions trajectories are globally bounded and, moreover, enter asymptotically a common subset of the phase space on which (1) is considered. Such situations, when the global attractor may occur only on some metric subspace of the starting phase space seem to be much more natural for parabolic problems and may frequently be observed. Hence, necessary and sufficient conditions for the existence of a restricted global attractor for (1) on a complete metric subspace of $X^{\alpha}$ (see Definition 3 of Section 2.1) will be studied here.

Certainly the heart of the paper are the examples studied in Section 3. We consider the Hodgkin-Huxley, Fitzhugh-Nagumo and LotkaVolterra systems of reaction-diffusion equations, 2-D Navier-Stokes and Burgers equations of hydrodynamics and mention the pattern formation equations as the Cahn-Hilliard model of alloy decomposition. These problems can be found in many earlier publications. One may refer to [CCS], 
[SM], [RO], [TE], [CRF], [GM], [CD1] [RS]. The main task of this paper is to give the unified approach to the global solvability and existence of global attractors for second and higher order parabolic problems mentioned above.

\section{General theory}

Section 2.0 contains two general theorems justifying existence of global attractors for semigroups generated by evolutionary equations with sectorial operators. It forms a basis for, specified in the case of parabolic systems, theorems of Section 2.1 where (1) is treated abstractly as a Cauchy problem in $\left[L^{p}(\Omega)\right]^{d}$. We study there a local semiflow $\{T(t)\}$ generated by (1) on fractional power spaces $X^{\alpha}$ and formulate an equivalent condition for distinguishing from a phase space $X^{\alpha}$ a subset $V$ for which a family of mappings $T(t): V \rightarrow V(t \geq 0)$ is a semiflow of global solutions of (1) and, in addition, has an attractor in $\mathrm{V}$.

\subsection{Background}

As a consequence of Assumption A-I the operator $\mathcal{A}_{p}$ defined in $\left[L^{p}(\Omega)\right]^{d}$ by $\mathcal{A}$ from (1) on the domain $\mathcal{D}\left(\mathcal{A}_{p}\right)=\left\{\phi \in\left[W^{2 m, p}(\Omega)\right]^{d}: \mathcal{B}_{j} \phi=0, j=\right.$ $1, \ldots, m d$ on $\partial \Omega\}(p>1)$ generates an analytic semigroup of linear operators $e^{-t \mathcal{A}_{p}}:\left[L^{p}(\Omega)\right]^{d} \rightarrow\left[L^{p}(\Omega)\right]^{d}, t \geq 0$ (cf. [AM, Chapt. I, Th. 2.4]). Hence, let us consider (1) as a Cauchy problem:

$$
\left\{\begin{array}{l}
\dot{u}+A_{p} u=F_{p}(u), \quad t>0, \\
u(0)=u_{0},
\end{array}\right.
$$

with $A_{p}=\mathcal{A}_{p}+k_{0} I d$ sectorial and positive in $\left[L^{p}(\Omega)\right]^{d}$ (so that $\operatorname{Re}\left(\sigma\left(A_{p}\right)\right) \geq$ $a>0$; in addition as a result of [TR, Th. 5.5.1] $A_{p}$ has compact resolvent) and $F_{p}$ a nonlinear substitution operator in $\left[L^{p}(\Omega)\right]^{d}$ induced by $f+k_{0} I d$; i.e.

$$
F(v)(x)=f\left(x, v(x), D^{1} v(x), \ldots, D^{k} v(x)\right)+k_{0} v(x) .
$$

Following [HA, p. 72], [HE, Chapt. 3]:

Definition 1 By a $D\left(A_{p}^{\alpha}\right)$-solution of (1) we understand a continuous function $u:\left[0, \tau_{u_{0}}\right) \rightarrow D\left(A_{p}^{\alpha}\right)$ satisfying $(2)$, such that $u_{t}:\left(0, \tau_{u_{0}}\right) \rightarrow$ $\left[L^{p}(\Omega)\right]^{d}$ is continuous and $u(t)$ belongs to $D\left(A_{p}\right)$ for each $t \in\left(0, \tau_{u_{0}}\right)$.

Results presented in Section 2.1 contain concretizations, for the case of 
systems of parabolic equations, of the two theorems stated below. Theorem 3 and Corollary 3 of Section 4 are derived from Proposition 1 and the idea of extending the properties of a semigroup on $X^{\beta}$ onto semigroups on $X^{\alpha}$ with $\alpha \in[\beta, 1)$ is exploited in all the theorems of Section 2.1. Condition (4) in Proposition 2 is also important, since it determines the choice of parameters $\alpha_{0}, p_{0}$ in such a way that the semigroup acting on $D\left(A_{p_{0}}^{\alpha_{0}}\right)$ and smoother spaces $D\left(A_{p}^{\alpha}\right)$ is automatically continuous in the $\left[W^{k, \infty}(\Omega)\right]^{d}$ norm, which immediately ensures Lipschitz continuity on bounded sets of the nonlinear term $F_{p}$ (cf. the comments in [C], [CRF], [CD2] and [CD3]).

The following result comes from Hale $[\mathrm{HA}]$ :

Proposition 1 Let $X$ be a complete metric space and $T(t): X \rightarrow X$, $t \geq 0$, be a compact $C^{0}$-semigroup. Assume that $\{T(t)\}$ is point dissipative; then, there is a global attractor for $\{T(t)\}$ in $X$.

Proof. The proof of this is very simple and follows from the fact that $\{T(t)\}$ is compact, from the continuity of the map $\left(t, u_{0}\right) \rightarrow T(t) u_{0}$ and from the point dissipativeness of $\{T(t)\}$.

As seen above, an important step is to guarantee compactness of the semigroup $\{T(t)\}$. In parabolic problems of the form (2), this is usually done by checking that $A_{p}$ has compact resolvent, proving that the associated semigroup is bounded and then using the variation of constants formula to obtain its compactness (cf. [HA, Sec. 4.2]). Another approach is presented in the following theorem.

Let $1>\alpha>\beta>0$ and assume that $A_{p}$ has compact resolvent. Assume also that the nonlinearity $F_{p}$ in $(2)$ is such that

$$
F_{p}: X^{\beta} \rightarrow X
$$

is a well defined map which is Lipschitz continuous on bounded subsets of $X^{\beta}$. Therefore $F_{p}: X^{\alpha} \rightarrow X$ is also Lipschitz continuous on bounded subsets of $X^{\alpha}$. Assume that solutions to (2) with initial data in $X^{\beta}$ are globally defined and let $\left\{T_{\beta}(t)\right\}$ denote the semigroup associated with (2) in $X^{\beta}$. Assume that $\left\{T_{\beta}(t)\right\}$ is point dissipative. Then the following result holds:

Theorem 1 Under assumptions as stated the semigroup $\left\{T_{\alpha}(t)\right\}$ associated with (2) in $X^{\alpha}$ is globally defined and bounded dissipative. Furthermore, there is a global attractor for $\left\{T_{\alpha}(t)\right\}$ in $X^{\alpha}$. 
Proof. Let $B$ be a bounded subset of $X^{\alpha}$ and let $B_{0} \subset X^{\beta}$ be such that $B_{0}$ attracts points of $X^{\beta}$ under the semigroup $\left\{T_{\beta}(t), t \geq 0\right\}$. Let us first prove that the semigroup $\left\{T_{\alpha}(t)\right\}$ is globally defined. If $u_{0} \in X^{\alpha}$ then $u_{0} \in X^{\beta}$ and $\left\{T_{\beta}(t) u_{0}, t \geq 0\right\}$ is a bounded subset of $X^{\beta}$ and therefore $\left\{F_{p}\left(T_{\beta}(t) u_{0}\right)\right\}$ is a bounded subset of $X$. Thus, omitting subscripts $\alpha, \beta$ wherever they are inessential, we have:

$$
\begin{aligned}
& \left\|T(t) u_{0}\right\|_{X^{\alpha}} \\
& \leq M e^{-a t}\left\|u_{0}\right\|_{X^{\alpha}}+\int_{0}^{t} M e^{-a(t-s)}(t-s)^{-\alpha}\left\|F_{p}\left(T(s) u_{0}\right)\right\|_{X} d s \\
& \leq M\left\|u_{0}\right\|_{X^{\alpha}}+M \sup _{t \geq 0}\left\|F_{p}\left(T(t) u_{0}\right)\right\|_{X} \int_{0}^{\infty} e^{-a \theta} \theta^{-\alpha} d \theta \\
& \quad=M\left\|u_{0}\right\|_{X^{\alpha}}+M \sup _{t \geq 0}\left\|F_{p}\left(T(t) u_{0}\right)\right\|_{X} \frac{\Gamma(1-\alpha)}{a^{1-\alpha}},
\end{aligned}
$$

which proves that the semigroup is globally defined on $X^{\alpha}$. Since $N\left(u_{0}\right):=$ $\sup _{t \geq 0}\left\|F_{p}\left(T_{\alpha}(t) u_{0}\right)\right\|_{X}$ is not necessarily a bounded function of $u_{0}$ we may not yet conclude that the semigroup $\left\{T_{\alpha}(t)\right\}$ is locally bounded; that is, for any bounded set $B \subset X^{\alpha}$ and $T>0$ the set $\left\{T(t) u_{0}: 0 \leq t \leq T, u_{0} \in B\right\}$ is bounded in $X^{\alpha}$. For that we use the following argument.

Note that $B$ viewed as a subset of $X^{\beta}$ is a precompact set and from the fact $\left(t, u_{0}\right) \rightarrow T_{\beta}(t) u_{0}$ from $R^{+} \times X^{\beta}$ to $X^{\beta}$ is continuous it follows that $\left\{T(t) u_{0}: 0 \leq t \leq T, u_{0} \in B\right\}$ is a bounded subset of $X^{\beta}$. Using the variation of constants formula (as before), we conclude that $\left\{T_{\alpha}(t)\right\}$ is locally bounded and therefore compact. To see that it is point dissipative we proceed as follows. We know that $\left\{T_{\beta}(t)\right\}$ is point dissipative; thus, for any $u_{0} \in X^{\alpha}$ there is a $t_{u_{0}}$ such that $\operatorname{dist}_{X^{\beta}}\left(T_{\beta}(t) u_{0}, B_{0}\right)<1$ for $t \geq t_{u_{0}}$. Thus, for such $u$ and $t$, we have

$$
T_{\alpha}(t) u=e^{-A_{p}\left(t-t_{u_{0}}\right)} T_{\alpha}\left(t_{u_{0}}\right) u+\int_{t_{u_{0}}}^{t} e^{-A_{p}(t-s)} F_{p}\left(T_{\alpha}(s) u\right) d s
$$

and, because of the smoothing action of $T(t)$ (cf. [HE, pp. 26, 57], [AM, (10), p. 59]),

$$
\begin{aligned}
\left\|T_{\alpha}(t) u\right\|_{X^{\alpha}} \leq M( & \left.t-t_{u_{0}}\right)^{\beta-\alpha} e^{-a\left(t-t_{u_{0}}\right)}\left\|T\left(t_{u_{0}}\right) u\right\|_{X^{\beta}} \\
& \quad+\int_{t_{u_{0}}}^{t} M e^{-a(t-s)}(t-s)^{-\alpha}\left\|F_{p}\left(T_{\alpha}(s) u\right)\right\|_{X} d s
\end{aligned}
$$




$$
\begin{gathered}
\leq M\left(t-t_{u_{0}}\right)^{\beta-\alpha} e^{-a\left(t-t_{u_{0}}\right)}\left(\sup _{v \in B_{0}}\|v\|_{X^{\beta}}+1\right) \\
+M \frac{\Gamma(1-\alpha)}{a^{1-\alpha}} \sup _{\operatorname{dist}_{X^{\beta}}\left(v, B_{0}\right)<1}\|F(v)\|_{X},
\end{gathered}
$$

which proves that $\left\{T_{\alpha}(t)\right\}$ is point dissipative and the proof is completed applying Proposition 1.

\subsection{Abstract approach to parabolic systems}

We start this section with the definition:

Definition 2 A $D\left(A_{p}^{\alpha}\right)$-solution $u\left(t, u_{0}\right)$ of (1) does not 'blow-up' iff $\tau_{\max }\left(u_{0}\right)=\infty$ and

$$
\sup _{t \in[0, \infty)}\left\|u\left(t, u_{0}\right)\right\|_{D\left(A_{p}^{\alpha}\right)}<\infty
$$

i.e. a solution $u\left(t, u_{0}\right)$ is defined globally in time and its trajectory is bounded.

The following is a well known result (cf. [HA, Sec. 2.2], [HE, Chapt. 3]):

Proposition 2 For $p_{0} \in(1, \infty)$ and $\alpha_{0} \in(0,1)$ satisfying

$$
k<2 m \alpha_{0}-\frac{n}{p_{0}}
$$

the nonlinear term $F: D\left(A_{p_{0}}^{\alpha_{0}}\right) \rightarrow\left[L^{p_{0}}(\Omega)\right]^{d}$ is Lipschitz continuous on bounded sets and a local semiflow $\{T(t)\}\left(T(t) u_{0}=u\left(t, u_{0}\right)\right.$ for $t \in$ $\left.\left[0, \tau_{\max }\left(u_{0}\right)\right)\right)$ of maximal $D\left(A_{p_{0}}^{\alpha_{0}}\right)$-solutions of $(1)$ is defined on $D\left(A_{p_{0}}^{\alpha_{0}}\right)$.

As a consequence of Definition 2, whenever $u_{0} \in D\left(A_{p_{0}}^{\alpha_{0}}\right)$ is such that $T(t) u_{0}$ does not 'blow-up', the estimate:

$$
\sup _{t>0}\left\|T(t) u_{0}\right\|_{Y} \leq \text { const }
$$

holds with $Y:=D\left(A_{p_{0}}^{\alpha_{0}}\right)$. However, even if (5) holds for some $u_{0} \in D\left(A_{p_{0}}^{\alpha_{0}}\right)$ and $Y \supset D\left(A_{p_{0}}\right)$ one cannot expect, in general, that the corresponding $D\left(A_{p_{0}}^{\alpha_{0}}\right)$-solution $T(t) u_{0}$ does not 'blow-up', unless simultaneously $F_{p}$ is subordinated to some power of $A_{p}$. More precisely:

Theorem 2 Let $p_{0}, \alpha_{0}$ satisfy (4) and $u_{0} \in D\left(A_{p_{0}}^{\alpha_{0}}\right)$. Then $T(t) u_{0}$ does not 'blow-up' if, and only if, there is a Banach space $Y \supset D\left(A_{p_{0}}\right)$ and a 
nondecreasing function $g:[0, \infty) \rightarrow[0, \infty)$ such that:

$$
\exists_{c>0} \forall_{t \in\left(0, \tau_{\max }\left(u_{0}\right)\right)}\left\|T(t) u_{0}\right\|_{Y} \leq c
$$

and

$$
\begin{aligned}
\exists_{\theta \in[0,1)} & \forall_{t \in\left(0, \tau_{\max }\left(u_{0}\right)\right)}\left\|F\left(T(t) u_{0}\right)\right\|_{\left[L^{p_{0}}(\Omega)\right]^{d}} \\
& \leq g\left(\left\|T(t) u_{0}\right\|_{Y}\right)\left(1+\left\|A_{p_{0}}^{\alpha_{0}} T(t) u_{0}\right\|_{\left[L^{\left.p_{0}(\Omega)\right]^{d}}\right.}^{\theta} .\right.
\end{aligned}
$$

Proof. As shown in [CD3, Th. 1], conditions (6) and (7) are sufficient to verify that $T(t) u_{0}$ does not 'blow-up'. To prove that (6) and (7) are also necessary observe that (6) follows from the boundedness of $\left\{T(t) u_{0}, t \geq 0\right\}$ in $D\left(A_{p_{0}}^{\alpha_{0}}\right)=: Y$. Furthermore, since

$$
\left\{T(t) u_{0}, t \geq 0\right\} \underset{\text { bounded }}{\subset} D\left(A_{p_{0}}^{\alpha_{0}}\right) \underset{\text { cont. }}{\subset}\left[C^{k}(c l \Omega)\right]^{d},
$$

then for any $t \geq 0$ the set

$$
B_{t}:=\left\{\left(x, T(t) u_{0}(x), D^{1} T(t) u_{0}(x), \ldots, D^{k} T(t) u_{0}(x)\right), x \in c l \Omega\right\}
$$

is bounded in $c l \Omega \times R^{d_{0}}$ (here $d_{0}=d \sum_{|\alpha| \leq k} 1$ ). More precisely:

$$
B_{t} \subset c l \Omega \times B_{R^{d_{0}}}\left(0, C\left\|T(t) u_{0}\right\|_{D\left(A_{p_{0}}^{\alpha_{0}}\right)}\right),
$$

where $B_{R^{d_{0}}}$ is a ball in $R^{d_{0}}$ centered in 0 with radius $C\left\|T(t) u_{0}\right\|_{D\left(A_{p_{0}}^{\alpha_{0}}\right)}$ and $C$ is the Sobolev constant from the embedding (8). Then by the continuity of $f$, for each fixed $t>0$ we verify that:

$$
\begin{aligned}
\| F_{p_{0}}( & \left.T(t) u_{0}\right) \|_{\left[L^{p}(\Omega)\right]^{d}} \\
\leq & |\Omega|^{\frac{1}{p_{0}}} \| f\left(\cdot, T(t) u_{0}(\cdot), D^{1} T(t) u_{0}(\cdot), \ldots, D^{k} T(t) u_{0}(\cdot)\right) \\
& +k_{0} T(t) u_{0}(\cdot) \|_{\left[L^{\infty}(\Omega)\right]^{d}} \\
\leq & |\Omega|^{\frac{1}{p_{0}}} \sup _{\substack{x \in c l \Omega \\
\left(p_{1}, \ldots, p_{d_{0}}\right) \in B_{t}}}\left\{\left|f\left(x, p_{1}, \ldots, p_{d_{0}}\right)\right|+k_{0}\left|\left(p_{1}, \ldots, p_{d}\right)\right|\right\} \\
\leq & |\Omega|^{\frac{1}{p_{0}}} \quad \sup _{x \in c l \Omega} \quad\left\{\left|f\left(x, p_{1}, \ldots, p_{d_{0}}\right)\right|\right. \\
& \left.\quad+k_{0}\left|\left(p_{1}, \ldots, p_{d}\right)\right|\right\}
\end{aligned}
$$

which proves that $(7)$ is satisfied with arbitrarily chosen $\theta \in[0,1)$ and a 
function $g:[0, \infty) \rightarrow[0, \infty)$ defined by

$$
g(y)=|\Omega|^{\frac{1}{p_{0}}} \sup _{\substack{x \in c l \Omega \\\left|\left(p_{1}, \ldots, p_{d_{0}}\right)\right| \leq C y}}\left\{\left|f\left(x, p_{1}, \ldots, p_{d_{0}}\right)\right|+k_{0}\left|\left(p_{1}, \ldots, p_{d}\right)\right|\right\} .
$$

Since the definition of $g$ is independent of $u_{0}$ then, as a consequence of Theorem 2, we get immediately:

Corollary 1 Let $p_{0}, \alpha_{0}$ satisfy (4) and $V \subset D\left(A_{p_{0}}^{\alpha_{0}}\right)$. Then $T(t) u_{0}$ does not 'blow-up' for $u_{0} \in V$ if, and only if, there is a Banach space $Y \supset D\left(A_{p_{0}}\right)$ and a nondecreasing function $g:[0, \infty) \rightarrow[0, \infty)$ such that:

$$
\forall_{u_{0} \in V} \exists_{c\left(u_{0}\right)>0} \forall_{t \in\left(0, \tau_{\max }\left(u_{0}\right)\right)}\left\|T(t) u_{0}\right\|_{Y} \leq c\left(u_{0}\right)
$$

and

$$
\begin{aligned}
\exists_{\theta \in[0,1)} \forall_{u_{0} \in V} & \forall_{t \in\left(0, \tau_{\max }\left(u_{0}\right)\right)}\left\|F\left(T(t) u_{0}\right)\right\|_{\left[L^{p_{0}}(\Omega)\right]^{d}} \\
& \leq g\left(\left\|T(t) u_{0}\right\|_{Y}\right)\left(1+\left\|A_{p_{0}}^{\alpha_{0}} T(t) u_{0}\right\|_{\left[L^{p_{0}}(\Omega)\right]^{d}}^{\theta}\right) .
\end{aligned}
$$

Corollary 1 contains criteria for boundedness of trajectories of points. In Proposition 3 below, a similar result concerning orbits of bounded sets is given.

Proposition 3 Let $p_{0}, \alpha_{0}$ satisfy (4) and $V \subset D\left(A_{p_{0}}^{\alpha_{0}}\right)$. Then the following conditions are equivalent:

$T(t) u_{0}$ does not 'blow-up' for $u_{0} \in V$ and for each bounded subset $B$ of $D\left(A_{p_{0}}^{\alpha_{0}}\right)$ the positive orbit $\{T(t) B \cap V, t \geq 0\}$ is bounded in $D\left(A_{p_{0}}^{\alpha_{0}}\right)$,

There is a Banach space $Y \supset D\left(A_{p_{0}}\right)$ and a nondecreasing function $g:[0, \infty) \rightarrow[0, \infty)$ such that $(10)$, (11) holds with the function $c: V \rightarrow[0, \infty)$ in (10) taking bounded subsets of $V$ into bounded subsets of $[0, \infty)$.

Proof. Sufficiency of (13) follows from [CD3, Th.1]. To prove that (13) is also necessary we only need to justify that $c\left(u_{0}\right)$ is bounded on bounded subsets of $V$ (cf. Cor. 1). Since $T(t) u_{0}$ does not 'blow-up' for $u_{0} \in V$ then all 
considerations of the proof of Th. 2 may be repeated until the nondecreasing function $g$ is defined as the condition (9) states. Thus, using (9), we get for each $t \geq 0$ :

$$
\begin{aligned}
& \left\|T(t) u_{0}\right\|_{D\left(A_{p_{0}}^{\alpha_{0}}\right)} \\
& \quad \leq C e^{-a t}\left\|u_{0}\right\|_{D\left(A_{p_{0}}^{\alpha_{0}}\right)}+\int_{0}^{t} C \frac{e^{-a s}}{s^{\alpha_{0}}} g\left(\sup _{s \in[0, t)}\left\|T(s) u_{0}\right\|_{D\left(A_{p_{0}}^{\alpha_{0}}\right)}\right) d s \\
& \quad \leq C\left\|u_{0}\right\|_{D\left(A_{p_{0}}^{\alpha_{0}}\right)}+g\left(\sup _{s \in[0, \infty)}\left\|T(s) u_{0}\right\|_{D\left(A_{p_{0}}^{\alpha_{0}}\right)}\right) C \frac{\Gamma\left(1-\alpha_{0}\right)}{a^{1-\alpha_{0}}} .
\end{aligned}
$$

Defining now for each $u_{0} \in V$

$$
c\left(u_{0}\right):=C\left\|u_{0}\right\|_{D\left(A_{p_{0}}^{\alpha_{0}}\right)}+C \frac{\Gamma\left(1-\alpha_{0}\right)}{a^{1-\alpha_{0}}} g\left(\sup _{s \in[0, \infty)}\left\|T(s) u_{0}\right\|_{D\left(A_{p_{0}}^{\alpha_{0}}\right)}\right),
$$

it is clear that if for each bounded subset $B$ of $D\left(A_{p_{0}}^{\alpha_{0}}\right)$ the orbit $\{T(t) B \cap$ $V, t \geq 0\}$ is bounded in $D\left(A_{p_{0}}^{\alpha_{0}}\right)$, then the function $c: V \rightarrow[0, \infty)$ given in (15) is bounded on bounded subsets of $V$.

As a result of [CD3, Th. 1] it follows that point dissipativeness of $\{T(t)\}$ is equivalent to the asymptotic independence of some introductory estimate of initial conditions. Connecting the mentioned result with Cor. 1 we obtain as a consequence:

Corollary 2 Let $p_{0}, \alpha_{0}$ satisfy (4) and $V \subset D\left(A_{p_{0}}^{\alpha_{0}}\right)$. Then the following two conditions are equivalent:

- $T(t) u_{0}$ does not 'blow-up' for $u_{0} \in V$ and there is a bounded subset $B$ of $D\left(A_{p_{0}}^{\alpha_{0}}\right)$ such that for each $u_{0} \in V$, dist ${ }_{D\left(A_{p_{0}}^{\alpha_{0}}\right)}\left(T(t) u_{0}, B\right) \rightarrow 0$ when $t \rightarrow+\infty$,

- There is a Banach space $Y \supset D\left(A_{p_{0}}\right)$ and a nondecreasing function $g:[0, \infty) \rightarrow[0, \infty)$ such that $(10),(11)$ hold and the estimate $(10)$ is asymptotically independent of $u_{0} \in V$, i.e.

$$
\exists c_{1}>0 \forall u_{u_{0} \in V} \limsup _{t \rightarrow+\infty}\left\|T(t) u_{0}\right\|_{Y}<c_{1} .
$$

After these preparations, the equivalent condition for existence of a restricted global attractor for $\{T(t)\}$ generated by (1) on a subset of $D\left(A_{p_{0}}^{\alpha_{0}}\right)$ may easily be formulated. As is seen in Definition 3 below, we shall consider 
further only semiflows which take bounded sets onto bounded sets.

Definition 3 Let $\{T(t)\}$ be a local semiflow on $D\left(A_{p_{0}}^{\alpha_{0}}\right)$. We say that $\mathcal{S} \subset D\left(A_{p_{0}}^{\alpha_{0}}\right)$ is a restricted global attractor for $\{T(t)\}$ in $D\left(A_{p_{0}}^{\alpha_{0}}\right)$ iff for some closed nonempty subset $V$ of $D\left(A_{p_{0}}^{\alpha_{0}}\right), T(t): V \rightarrow V, t \geq 0$, is a global semiflow on $V$ such that positive orbits of bounded sets are bounded and $\mathcal{S}$ is a global attractor for $\{T(t)\}$ restricted to $\mathrm{V}$ in the sense of the definition stated in [HA, Sec. 3.4].

Remark 1. When $V=D\left(A_{p_{0}}^{\alpha_{0}}\right)$, Definition 3 coincides with the original definition of the global attractor contained in Hale's monograph [HA]. However, the above notion of a restricted global attractor seems to be more useful in applications. As seen in many examples, although (1) (under an appropriate choice of parameters $\alpha$ and $p$ ) almost always generates a local semiflow on $D\left(A_{p}^{\alpha}\right)$, it is rather rare for it to keep trajectories of all points from $D\left(A_{p}^{\alpha}\right)$ bounded in time, or even if this is so, it is much more rare that this semiflow is point dissipative on the whole phase space $D\left(A_{p}^{\alpha}\right)$. Hence, the results presented here give equivalent conditions for selecting in $D\left(A_{p}^{\alpha}\right)$ a subset $V$ such that $\{T(t)\}$ restricted to $V$ is globally defined and point dissipative; i.e. there is a restricted global attractor for $\{T(t)\}$ (see Theorem 3 below).

Remark 2. In [HA, p. 17] a local attractor was defined as a compact invariant set attracting an open neighborhood of itself. This notion is, in general, different from the notion introduced above of a restricted global attractor unless a special form of $V$ is considered. For instance, in the well known Cahn-Hilliard equation (see [CD2], [TE] for details) the set $V$ has the form:

$$
V=\left\{\phi \in D\left(A_{p}^{\alpha}\right) ;|\bar{\phi}| \leq b\right\}, b>0 \text { where } \bar{\phi}:=|\Omega|^{-1} \int_{\Omega} \phi(x) d x .
$$

Clearly $V$ is unbounded in $D\left(A_{p}^{\alpha}\right)$ and among elements of a restricted global attractor $\mathcal{A}_{V}$ (cf. [TE], [CD2]) are constant stationary solutions:

$$
\psi(x) \equiv|\Omega|^{-1} a, \text { for } a \in[0, b] .
$$

Since any open neighbourhood of $\mathcal{A}_{V}$ in $D\left(A_{p}^{\alpha}\right)$ must contain element $\phi_{\varepsilon}$ with spatial average $\bar{\phi}_{\varepsilon}=b+\varepsilon(\varepsilon>0)$ and spatial averages of solutions of the Cahn-Hilliard equation are preserved in time, then $\mathcal{A}_{V}$ will not attract the trajectory of $\phi_{\varepsilon}$ so that $\mathcal{A}_{V}$ is not a local attractor in the sense of [HA, p. 17] in $D\left(A_{p}^{\alpha}\right)$. 
Connecting Cor. 1, Prop. 3, Th. 2 and using the results of [HA, Chapt. $3,4]$ we obtain finally:

Theorem 3 Let $p_{0}, \alpha_{0}$ satisfy $(4),\{T(t)\}$ be a local semiflow on $D\left(A_{p_{0}}^{\alpha_{0}}\right)$ introduced in Prop. 2 and the resolvent of $A_{p_{0}}$ be compact. Then $\{T(t)\}$ has a restricted global attractor in $D\left(A_{p_{0}}^{\alpha_{0}}\right)$ if, and only if, the conjunction of conditions (13) and (16) holds with some closed and positively invariant nonempty subset $V$ of $D\left(A_{p_{0}}^{\alpha_{0}}\right)$.

Based on the results given above, in the next section a number of parabolic problems is studied. Finally, in Section 4, the above abstract requirements are translated into algebraic conditions concerning the data of problem (1).

\section{Examples}

\subsection{Dissipation Through Contracting Rectangles}

We start with the general setting that was formulated and studied by C. C. Conley, J. A. Smoller and their collaborators (see e.g. [CCS], [SM]).

In general, showing that a semigroup $\{T(t)\}$ is point dissipative is done with the use of La Salle's invariance theory and though it may be a delicate step, it can be accomplished in many applications (see, for example [C] or [CRF]).

Let $\Omega$ be a bounded domain in $R^{n}, n \leq 3$, with smooth boundary and $D$ denotes a positive definite diagonal matrix. Consider the following second order semilinear parabolic system:

$$
\left\{\begin{array}{l}
u_{t}=D \Delta u+f(u), \quad x \in \Omega, t>0 \\
\frac{\partial u}{\partial N}=0, \quad x \in \partial \Omega, t>0
\end{array}\right.
$$

where $f: \mathcal{M} \subset R^{d} \rightarrow R^{d}$ is a locally Lipschitz continuous function and $\mathcal{M}$ is a closed rectangle in $R^{n}$ such that in every face of the rectangle

$$
f(u) \cdot N(u) \leq 0
$$

with $N(u)$ denoting the outward normal vector to the corresponding face. Assume that $\alpha>\frac{n}{4}$ and let $V=\left\{u \in X^{\alpha}: \forall_{x \in \Omega} u(x) \in \mathcal{M}\right\}$. It has been shown in [C] that (17) is locally well posed in $V$. In addition to the above hypothesis, assume that there is a bounded rectangle $R_{0}$ containing 0 and 
a point $x_{0} \in \mathcal{M}$ with

$$
R_{t}=x_{0}+t R_{0}
$$

such that $\left\{R_{t}, t \geq 0\right\}$ covers $\mathcal{M}$ and

$$
f(u) \cdot N(u)<0
$$

for any $u$ in a closed face of $R_{t} \cap \mathcal{M}$ which is not contained in a face of $\mathcal{M}$, $N(u)$ being the outward normal vector to that face.

Remark 3. Even though the above conditions seem complicated at first, they are very natural. They merely state that the flow points inward in a family of rectangles (larger then $R_{0}$ ) which cover $\mathcal{M}$. And with Neumann boundary conditions this is expected to occur in many examples for which one hopes to prove existence of global attractors since the solutions to $\dot{u}=$ $f(u)$ are also solutions to the problem (17).

Next we describe how the above conditions imply dissipativeness in $X^{\alpha}$. Let $P_{R_{0}}: \mathcal{M} \rightarrow R^{+}$be the function defined by

$$
P_{R_{0}}(u)=\inf \left\{t \geq 0: u \in R_{t}\right\}
$$

and $F_{R_{0}}: V \rightarrow R^{+}$be the function defined by

$$
F_{R_{0}}(w)=\sup _{x \in \Omega} P_{R_{0}}(w(x)) .
$$

Note that since $X^{\alpha} \subset\left[L^{\infty}(\Omega)\right]^{d}$ we have that $F_{R_{0}}$ is a Lipschitz function in bounded subsets of $V$. It has been proved in [C] that

$$
\dot{F}_{R_{0}}(u)<-\eta
$$

for any $u_{0}$ whose image is not contained in $R_{0}$. Here $\dot{F}_{R_{0}}$ denotes the derivative along solutions of (17). Therefore, $F_{R_{0}}$ is a Lyapunov function and from La Salle's invariance theory we obtain that $T_{Y}(t)$ is globally defined and point dissipative in the uniform norm, proving that (16) is satisfied. Also, using invariant regions and the variation of constants formula, one easily obtains boundedness (see [C]) and from Proposition 2, (13) is satisfied. Theorem 3 guarantees the existence of a restricted global attractor in $V$.

Remark 4. An alternative way to get point dissipativeness is to use point dissipativeness in the uniform norm to obtain dissipativeness in $V$ through 
the variation of constants formula (see $[\mathrm{C}]$ ) and then use the previous comments to obtain boundedness in $V$.

For easier studies of several physical models the following short introduction is useful. Let $\Omega$ be a bounded smooth subset of $R^{n}, X=\left[L^{2}(\Omega)\right]^{d}$, and $A: D(A) \subset X \rightarrow X$ be the unbounded product operator defined by

$$
-A=[-\Delta+\delta I d] \times \ldots \times[-\Delta+\delta I d]
$$

on the domain

$$
D(A)=\left\{u \in\left[H^{2}(\Omega)\right]^{d}: \frac{\partial u_{i}}{\partial N}=0,1 \leq i \leq d \text { in } \partial \Omega\right\} .
$$

The operator $-A$ is selfadjoint, positive with compact resolvent and we can define its fractional powers $(-A)^{\alpha}$ and the associated fractional power spaces $X^{\alpha}$ (cf. Proposition 4). It follows next from the fact that $A$ has compact resolvent that $X^{\alpha_{1}}$ is compactly embedded in $X^{\alpha_{2}}$ whenever $\alpha_{1}>$ $\alpha_{2}$. Also $X^{\alpha}=\left[H^{2 \alpha}(\Omega)\right]^{d}$ for $\alpha \leq \frac{1}{2}$ and $X^{\alpha} \subset\left[L^{\infty}(\Omega)\right]^{d}$ with compact embedding for $\alpha>\frac{n}{4}$.

\subsection{The Hodgkin-Huxley Equations}

Consider the system of weakly coupled semilinear parabolic partial differential equations in $\Omega \subset R^{n}, n \leq 3$,

$$
\left\{\begin{array}{l}
c u_{t}=C^{-1} \Delta u+g(u, v, w, z), \quad x \in \Omega, t>0, \\
v_{t}=\epsilon_{1} \Delta v+g_{1}(u)\left(h_{1}(u)-v\right), \quad x \in \Omega, t>0, \\
w_{t}=\epsilon_{2} \Delta w+g_{2}(u)\left(h_{2}(u)-w\right), \quad x \in \Omega, t>0, \\
z_{t}=\epsilon_{2} \Delta z+g_{3}(u)\left(h_{3}(u)-z\right), \quad x \in \Omega, t>0, \\
\frac{\partial u}{\partial N}=\frac{\partial v}{\partial N}=\frac{\partial w}{\partial N}=\frac{\partial z}{\partial N}, \quad x \in \partial \Omega, t>0,
\end{array}\right.
$$

where $C, c_{i}$ and $\epsilon_{i}, i=1,2,3$, are positive constants, and $g$ is defined by

$$
\begin{aligned}
g(u, v, w, z)= & k_{1} v^{3} w\left(c_{1}-u\right)+k_{2} z^{4}\left(c_{2}-u\right)+k_{3}\left(c_{3}-u\right) \\
& c_{1}>c_{3}>0>c_{2} .
\end{aligned}
$$

Furthermore, $g_{i}>0,1>h_{i}>0, i=1,2,3$. In this model the variables $v, w, z$ represent chemical concentrations, and are thus nonnegative, while $u$ denotes electric potential. The equations are a mathematical model for the physiological phenomenon of signal transmission across axons. 
The above system is locally well posed in $V=\left\{\left(u_{1}, u_{2}, u_{3}, u_{4}\right) \in X^{\alpha}\right.$ : $\left.\forall_{x \in \Omega} u_{1} \in R^{1}, u_{i}(x) \geq 0,2 \leq i \leq 4\right\}, \alpha>\frac{n}{4}$. In what follows we prove that the problem (19) has a restricted global attractor in $V$.

Under these hypotheses it is easy to see that (18) is satisfied for the vector field $F(u, v, w, z)=\left(g(u, v, w, z), g_{1}(u)\left(h_{1}(u)-v\right), g_{2}(u)\left(h_{2}(u)-w\right)\right.$, $\left.g_{3}(u)\left(h_{3}(u)-z\right)\right)^{\top}$ in $\mathcal{M}=\left\{\left(u_{1}, u_{2}, u_{3}, u_{4}\right) \in R^{4}: u_{i} \geq 0,2 \leq i \leq\right.$ $\left.4, u_{1} \in R^{1}\right\}$. Choose $x_{0}=(0,0,0,0)$ and $R_{0}=\left[-M_{1}, M_{1}\right] \times[0,1]^{3}, M_{1} \geq$ $\max \left\{c_{1},\left|c_{2}\right|\right\}$. It follows from the results of the previous sections that the problem (19) has a restricted global attractor in $V$.

\subsection{The Fitzhugh-Nagumo Equations}

Consider the equations

$$
\left\{\begin{array}{l}
u_{t}=\Delta u+f(u)-v, \quad x \in \Omega, t>0, \\
v_{t}=\epsilon \Delta v+\sigma u-\gamma v, \quad x \in \Omega, t>0, \\
\frac{\partial u}{\partial N}=\frac{\partial v}{\partial N}=0, \quad x \in \partial \Omega, t>0,
\end{array}\right.
$$

where $\sigma, \gamma$ and $\epsilon$ are positive constants. The function $f$ has the qualitative form of a cubic polynomial, for example $f(u)=-u(u-\beta)(u-1)$, where $0<\beta<\frac{1}{2}$. This system is known as the Fitzhugh-Nagumo Equations and also constitutes a mathematical model for transmission of nerve impulses in axons.

Therefore, the system (21) defines a local semigroup in $X^{\alpha}, \alpha>\frac{n}{4}$. If we choose a rectangle $R$ such that in the lower face $\sigma u-\gamma v>0$, in the upper face $\sigma u-\gamma v<0$, in the left face $f(u)-v>0$, in the right face $f(u)-v<0$, then (18) is satisfied. The fact that $f$ grows more than linearly guarantees that it is always possible to find such a rectangle and to justify the existence of a restricted global attractor for $(21)$ in $V_{R}=\left\{(u, v) \in X^{\alpha}\right.$ : $(u(x), v(x)) \in R, x \in \Omega\}, \alpha>\frac{n}{4}$.

\subsection{Superconductivity of Liquids}

Consider the following system of parabolic partial differential equations:

$$
\left\{\begin{array}{l}
u_{t}=D \Delta u+\left(1-|u|^{2}\right) f(u), \quad x \in \Omega, t>0, \\
\frac{\partial u}{\partial N}=0, \quad x \in \partial \Omega, t>0,
\end{array}\right.
$$

where $f:=\left(f_{1}, \ldots, f_{d}\right)$ is a locally Lipschitz function satisfying $f_{i}(u) u_{i}>0$ for $u_{i} \neq 0,1 \leq i \leq d, D$ is as before. Then, if $x_{0}=0$ and $R_{0}=[-1,1]^{d}$, the 
condition (18) is satisfied and the results of the previous sections imply the existence of a global attractor for the problem (22) in $X^{\alpha}, \alpha>\frac{n}{4}$.

\subsection{Lotka-Volterra Models for Two Competing Species}

Consider the following Lotka-Volterra Model in $X^{\alpha}$ for two species competing for the same food source, with each species having logistic growth in the absence of the other and taking into account the diffusion:

$$
\left\{\begin{array}{l}
u_{t}=d_{1} \Delta u+u(1-u-a v), \quad x \in \Omega, t>0, \\
v_{t}=d_{2} \Delta v+\rho v(1-v-b u), \quad x \in \Omega, t>0 \\
\frac{\partial u}{\partial N}=\frac{\partial v}{\partial N}=0, \quad x \in \partial \Omega, t>0
\end{array}\right.
$$

where $a, b, \rho$ are positive constants. Let $\mathcal{M}:=[0, \infty) \times[0, \infty)$ and $V=$ $\left\{\phi \in X^{\alpha} ; \forall_{x \in \Omega} \phi(x) \in \mathcal{M}\right\}, \alpha>\frac{n}{4}$. Note that

$$
\begin{aligned}
& u^{2}(1-u-a v)<0 \text { for } u>1, v \geq 0 \\
& \rho v^{2}(1-v-b u)<0 \text { for } v>1, u \geq 0 .
\end{aligned}
$$

Therefore, taking $x_{0}=0$ and $R_{0}=[0,1] \times[0,1]$, the results in the previous sections imply that the problem (23) has a restricted global attractor in $V$.

\subsection{Systems of parabolic equations in $\boldsymbol{H}^{1}(\Omega)$ in subcritical growth case without gradient structure}

The present example will show that although the abstract approach of Part 2 is convenient in applications, because of restrictions imposed e.g. by condition (4), separate treating of specific problems will sometimes extend the range of spaces in which global attractors could be detected. Such extension, however, requires extra assumptions imposed on nonlinearity.

Let $f: R^{d} \rightarrow R^{d}$ be a locally Lipschitz continuous function and assume that there are constants $\xi_{i}, 1 \leq i \leq d$, such that

$$
f_{i}(u) u_{i}<0
$$

for all $u \in R^{d}$ with $\left|u_{i}\right|>\xi_{i}$. Consider the system of parabolic problems:

$$
\left\{\begin{array}{l}
u_{t}=D \Delta u+f(u) \text { in } R^{+} \times \Omega, \\
\frac{\partial u}{\partial N}=0 \text { on } \partial \Omega, \\
u(0, x)=u_{0}(x), \quad x \in \Omega .
\end{array}\right.
$$


Choosing arbitrary $\alpha \in(0,1), p \in(1, \infty)$ satisfying (4);

$$
\alpha>\frac{n}{2 p}
$$

and any $u_{0} \in D\left(A_{p}^{\alpha}\right)$, as a direct consequence of the $\left[L^{\infty}(\Omega)\right]^{d}$ a priori estimates of $D\left(A_{p}^{\alpha}\right)$ solutions to (25) following from 3.1, we get an estimate

$$
\left\|f\left(u\left(t, u_{0}\right)\right)\right\|_{\left[L^{\infty}(\Omega)\right]^{d}} \leq g\left(\left\|u\left(t, u_{0}\right)\right\|_{\left[L^{\infty}(\Omega)\right]^{d}}\right),
$$

which is a special form of $(11)$ with $Y=\left[L^{\infty}(\Omega)\right]^{d}$. Restricting the semigroup generated by $(25)$ to $u_{0}$ with values in a positively invariant rectangle $\mathcal{M}$, from 3.1 we get automatically the existence of a restricted global attractor as a consequence of Theorem 3 with

$$
V=D\left(A_{p}^{\alpha}\right) \cap\left\{\phi \in\left[L^{\infty}(\Omega)\right]^{d} ; \phi(x) \in \mathcal{M}\right\} .
$$

Assume now additionally that $n=3$ and there are constants $\gamma<2$ and $C>0$ such that

$$
|f(u)-f(v)|^{2} \leq C\left(1+|u|^{2 \gamma}+|v|^{2 \gamma}\right)|u-v|^{2} .
$$

Our first aim will be to prove that the problem (25) is locally well posed in $X^{\alpha}$ for some $\alpha<\frac{1}{2}$. Then, we will prove that the solutions of (25) are globally defined and that the semigroup associated with (25) is point dissipative in $X^{\alpha}$. With that information we use the fact that $X^{\frac{1}{2}}$ is compactly embedded in $X^{\alpha}, \alpha<\frac{1}{2}$, to obtain that the semigroup associated with (25) has a global attractor in $X^{\frac{1}{2}}$. The following lemma guarantees that the problem (25) is locally well posed.

Lemma 1 The map $f^{e}:\left[H^{s}(\Omega)\right]^{d} \rightarrow\left[L^{2}(\Omega)\right]^{d}$, where $f^{e}(u)(x)=f(u(x))$ for $x \in \Omega$, is well defined and Lipschitz continuous on bounded subsets of $\left[H^{s}(\Omega)\right]^{d}$ for $s \geq \frac{3}{2}\left(1-\frac{1}{\gamma+1}\right)$.

Proof. It is enough to prove the above result for $s=\frac{3}{2}\left(1-\frac{1}{\gamma+1}\right)$. Let $r>0$ and $u, v \in\left[H^{s}(\Omega)\right]^{d}$ be such that $\|u\|_{\left[H^{s}(\Omega)\right]^{d}} \leq r$ and $\|v\|_{\left[H^{s}(\Omega)\right]^{d}} \leq r$. Then,

$$
\begin{aligned}
& \int_{\Omega}|f(u(x))-f(v(x))|^{2} d x \\
& \quad \leq C \int_{\Omega}\left(1+|u(x)|^{2 \gamma}+|v(x)|^{2 \gamma}\right)(u(x)-v(x))^{2} d x
\end{aligned}
$$




$$
\leq C\left(|\Omega|^{\frac{1}{q}}+\|u\|_{\left[L^{2 \gamma q}(\Omega)\right]^{d}}^{2 \gamma}+\|v\|_{\left[L^{2 \gamma q}(\Omega)\right]^{d}}^{2 \gamma}\|u-v\|_{\left[L^{2 q^{\prime}}(\Omega)\right]^{d}}^{2},\right.
$$

where $\frac{1}{q}+\frac{1}{q^{\prime}}=1$. Since $\left[H^{s}(\Omega)\right]^{d} \subset\left[L^{p}(\Omega)\right]^{d}$ with continuous inclusion for $s=\frac{3}{2}-\frac{3}{p}$, we impose that $2 q^{\prime}=p$. Then, $2 \gamma q=p$. Since $\left[H^{s}(\Omega)\right]^{d}$ is continuously embedded in $\left[L^{p}(\Omega)\right]^{d}$, we have that

$$
\begin{aligned}
& \left\|f^{e}(u)-f^{e}(v)\right\|_{\left[L^{2}(\Omega)\right]^{d}} \\
& \quad \leq c K\left(|\Omega|^{\frac{2 \gamma}{p}}+K^{2 \gamma}\left(\|u\|_{\left[H^{s}(\Omega)\right]^{d}}^{2 \gamma}+\|v\|_{\left[H^{s}(\Omega)\right]^{d}}^{2 \gamma}\right)\right)^{\frac{1}{2}}\|u-v\|_{\left[H^{s}(\Omega)\right]^{d}} \\
& \quad \leq c K\left(|\Omega|^{\frac{2 \gamma}{p}}+2 K^{2 \gamma} r^{2 \gamma}\right)^{\frac{1}{2}}\|u-v\|_{\left[H^{s}(\Omega)\right]^{d}},
\end{aligned}
$$

where $K$ is the embedding constant for $\left[H^{s}(\Omega)\right]^{d} \subset\left[L^{p}(\Omega)\right]^{d}$ and the result is proved.

Let $u_{0} \in X^{\alpha}, \alpha \geq \frac{3}{4}\left(1-\frac{1}{\gamma+1}\right)$. Then, from Lemma 1, there exists a solution $u\left(t, u_{0}\right)$ to the problem $(25)$ satisfying $u\left(0, u_{0}\right)=u_{0}$. This local solution is such that $u\left(t, u_{0}\right) \in X^{\beta}$, for every $\beta \leq 1$ and $t>0$ and for as long as it exists. Therefore, $u\left(t, u_{0}\right) \in\left[L^{\infty}(\Omega)\right]^{d}$ for $t>0$ and for as long as it exists. If $\left[0, t_{\max }\right)$ denotes the maximal interval of existence of this solution and $t_{0} \in\left(0, t_{\max }\right)$, we have that $u\left(t_{0}, u_{0}\right)(x) \in R_{\tau}$ for some $\tau>0$. Therefore, from the results in 3.1 we obtain that this solution does not blow up in finite time and as consequence of this, it must exist for all time. Also from the results in 3.1 we obtain that the semigroup associated with (25) is point dissipative in $\left[L^{\infty}(\Omega)\right]^{d}$. Using the variation of constants formula we obtain that this semigroup is point dissipative in $X^{\alpha}, \alpha \geq \frac{3}{4}\left(1-\frac{1}{\gamma+1}\right)$. Theorem 1 implies that (25) has a global attractor in $X^{\beta}, \beta>\frac{3}{4}\left(1-\frac{1}{\gamma+1}\right)$, in particular it has a global attractor in $X^{\frac{1}{2}}=\left[H^{1}(\Omega)\right]^{d}$ and the result is proved.

Remark 5. No gradient structure is assumed for (25). Thus, we have been able to prove the existence of global attractors in $\left[H^{1}(\Omega)\right]^{d}$ for systems of parabolic problems in the subcritical growth case without assuming that the nonlinearity has a symmetric Jacobian.

\subsection{Fluid flow problems}

3.7.1. 2D-Navier-Stokes system. The Navier-Stokes system was studied by many authors but we will refer here only to [HE, p. 79-81], 
[TE, p. 102-113] and [GM] for details or references. Let us consider

$$
\left\{\begin{array}{l}
u_{t}=\Delta u-(u, \nabla) u+f-\nabla p \text { in }(0, T) \times D \\
\operatorname{div} u=0, \text { in }(0, T) \times D \\
u=0 \text { on } S \times(0, T) \\
u(0, x)=u_{0}(x) \text { in } D
\end{array}\right.
$$

where $D$ is a bounded domain in $R^{2}$ with a smooth boundary $S, u=$ $\left(u^{1}(t, x), u^{2}(t, x)\right)$ denotes velocity, $p(t, x)$ pressure and $f=\left(f^{1}(x), f^{2}(x)\right)$ the external forces. Projecting this problem $\left(P_{r}\right)$ onto a subspace $X_{r} \subset$ $\left[L^{r}(D)\right]^{2}, 1<r<\infty$, of divergence-free functions

$$
X_{r}=\operatorname{cl}_{\left[L^{r}(D)\right]^{2}}\left\{\phi \in\left[C_{0}^{\infty}(D)\right]^{2} ; \operatorname{div} \phi=0\right\}
$$

we get (27) in an abstract form

$$
u_{t}+A_{r} u=F_{r} u+P_{r} f, \quad t>0, u(0)=u_{0},
$$

with sectorial matrix operator

$$
A_{r}=-P_{r}\left[\begin{array}{cc}
\Delta & 0 \\
0 & \Delta
\end{array}\right]
$$

(cf. [GM, Lem. 1.1], [HE, p. 80-81]) defined on the domain $D\left(A_{r}\right)=X_{r} \cap$ $\left\{\phi \in\left[W^{2, r}(D)\right]^{2} ;\left.\phi\right|_{\partial D}=0\right\}$ and nonlinear term $F_{r} u=-P_{r}(u, \nabla) u$. Compactness of the resolvent $\left(A_{r}+\lambda I d\right)^{-1}: X_{r} \rightarrow X_{r}, \lambda \in \sigma\left(A_{r}\right)$, follows from the estimate in [GM, Lem. 3.1] with $m=0$ and compactness of the embedding $\left[W^{2, r}(D)\right]^{2} \rightarrow\left[L^{r}(D)\right]^{2}$.

In order to fulfil condition $(4)$, we choose $p_{0} \in(1, \infty)$ and $\alpha_{0} \in(0,1)$ satisfying

$$
1<2\left(\alpha_{0}-\frac{1}{p_{0}}\right)
$$

From [GM, p. 269] for $0 \leq \alpha \leq 1$, it is known that

$$
D\left(A_{r}^{\alpha}\right)=\left[X_{r}, D\left(A_{r}\right)\right]_{\alpha}
$$

and, whenever $\alpha \geq 0, D\left(A_{r}^{\alpha}\right)$ is continuously embedded in the space of Bessel potentials $\left[H_{r}^{2 \alpha}(D)\right]^{2}$. In particular, for $\alpha \in\left[\alpha_{0}, 1\right)$ and $r \geq p_{0}, D\left(A_{r}^{\alpha}\right)$ is a subset of $\left[L^{\infty}(D)\right]^{2} \cap\left[H_{r}^{1}(D)\right]^{2}$ and the nonlinear term $F_{r}: D\left(A_{r}^{\alpha}\right) \rightarrow X_{r}$ is 
Lipschitz continuous on bounded sets. Indeed, for $\phi, \psi \in D\left(A_{r}^{\alpha}\right)$

$$
\begin{aligned}
& \left\|F_{r} \phi-F_{r} \psi\right\|_{X^{r}} \\
& \leq C_{r}\|\phi\|_{\left[W^{1, r}(D)\right]^{2}}\|\phi-\psi\|_{\left[W^{1, r}(D)\right]^{2}} \\
& \quad+C_{r}\|\phi-\psi\|_{\left[W^{1, r}(D)\right]^{2}}\|\psi\|_{\left[W^{1, r}(D)\right]^{2}} \\
& \leq C_{r} \max \left\{\|\phi\|_{\left[W^{1, r}(D)\right]^{2}},\|\psi\|_{\left[W^{1, r}(D)\right]^{2}}\right\}\|\phi-\psi\|_{\left[W^{1, r}(D)\right]^{2}}
\end{aligned}
$$

(cf. [GM, Lem. 3.2]), so that local solvability of (28) follows.

Now for $\alpha_{0}>\frac{1}{2}$ and $p_{0}>2$ (resulting from $\left[(29)\right.$ ), our $D\left(A_{r}^{\alpha}\right)$ solution $\left(\alpha \in\left[\alpha_{0}, 1\right), r \geq p_{0}\right)$ is automatically a solution in the sense of [TE, Th. 2.1, p. 106] and we are allowed to use an a priori estimate $(2.36)$ of $[\mathrm{TE}$, p. 109], to get

$$
\|u(t)\|_{\left[H_{0}^{1}(D)\right]^{2}} \leq \text { const, for } t \geq t_{0}+r
$$

with const independent of $u_{0}$ (note that estimate (2.25) of [TE] is too weak for our needs, since it corresponds to borderline values of $\gamma_{j}, j=0,1$ in (44)).

Based on [GM, Lem. 3.3] we can estimate the right side of (28) as follows:

$$
\begin{aligned}
\left\|F_{r} u+P_{r} f\right\|_{\left[L^{r}(D)\right]^{2}} & \leq\left\|P_{r}(u, \nabla) u\right\|_{\left[L^{r}(D)\right]^{2}}+C_{0, r}\|f\|_{\left[L^{r}(D)\right]^{2}} \\
& \leq C_{r}\|u\|_{\left[W^{1, r}(D)\right]^{2}}^{2}+C_{0, r}\|f\|_{\left[L^{r}(D)\right]^{2}} .
\end{aligned}
$$

Simple application of the Nirenberg-Gagliardo inequality [AM, p. 60] gives next:

$$
\|u\|_{\left[W^{1, r}(D)\right]^{2}} \leq c\|u\|_{\left[H_{0}^{1}(D)\right]^{2}}^{1-\theta}\|u\|_{D\left(A_{r}^{1-\varepsilon}\right)}^{\theta}
$$

$\left(\theta>\frac{1-\frac{2}{r}}{2-2 \varepsilon-\frac{2}{r}}\right.$ and $\varepsilon \in\left(0, \frac{1}{r}\right)$ is arbitrary, so that $\left.2 \theta<1\right)$. Hence, (31) may be extended to a suitable form of (11), and we obtain

$$
\begin{aligned}
& \left\|F_{r} u+P_{r} f\right\|_{\left[L^{r}(D)\right]^{2}} \\
& \quad \leq C^{2}\|u\|_{\left[H_{0}^{1}(D)\right]^{2}}^{2-2 \theta}\|u\|_{D\left(A_{r}^{1-\varepsilon}\right)}^{2 \theta}+C_{0, r}\|f\|_{\left[L^{r}(D)\right]^{2}},
\end{aligned}
$$

which together with (30) justifies the applicability of Corollary 2. Existence of a global attractor for the semigroup generated by $(27)$ on $D\left(A_{r}^{\alpha}\right)$ with $r>2$ and $\alpha \in\left(\max \left\{1-\frac{1}{r} ; \frac{1}{2}+\frac{1}{r}\right\}, 1\right)$ now follows from Theorem 3 . 
3.7.2. Burgers equation. A simpler physical model for viscous fluid motion is the Burgers equation [DL]:

$$
\left\{\begin{array}{l}
v_{t}=\nu v_{x x}+U v-\frac{1}{2}\left(v^{2}\right)_{x}, \quad t>0, x \in(0, \pi) \\
U_{t}=P-\nu U-\int_{0}^{\pi} v^{2}(t, x) d x, \quad t>0 \\
v(t, 0)=v(t, \pi)=0, \quad t>0 \\
v(0, x)=v_{0}(x), \quad x \in(0, \pi) \\
U(0)=U_{0}
\end{array}\right.
$$

where $\nu>0$ denotes viscosity and $P>0$ stays for a constant pressure drop.

Denoting $A_{1}=-\nu \frac{\partial^{2}}{\partial x^{2}}$ one can see that $\left(A_{1}, I d,(0, \pi)\right)$ is a regular parabolic initial boundary value problem (see part (i) of Prop. 4 in Section 4 ). Hence, $A_{1}$ is a sectorial operator in $L^{p}(0, \pi)$. Moreover $A_{2}:=-\nu I d$ with $D\left(A_{2}\right)=R^{1}$ (as bounded and linear; cf. [HE, Chapt. 1, Ex.1]) is also sectorial and hence, the problem (33) admits an abstract formulation (2) with the product operator $A_{p}:=A_{1} \times A_{2}$ considered on the domain $D\left(A_{p}\right)=$ $\left(W_{0}^{1, p}(0, \pi) \cap W^{2, p}(0, \pi)\right) \times R^{1}$ being sectorial in $L^{p}(0, \pi) \times R^{1}$ (cf. [HE, Chapt. 1, Ex. 3]). In this example the substitution operator $F_{p}$ is defined as:

$$
F_{p}(U, v):=\left(U v-\frac{1}{2}\left(v^{2}\right)_{x}, P-\int_{0}^{\pi} v^{2}(t, x) d x\right) .
$$

Whenever $2 \alpha_{0}-\frac{1}{p_{0}}>1$ it may be seen that $F_{p_{0}}$ acting from $D\left(A_{p_{0}}^{\alpha_{0}}\right)=$ $D\left(A_{1}^{\alpha_{0}} p_{0}\right) \times R^{1}$ into $L^{p_{0}}(0, \pi) \times R^{1}$ is Lipschitz continuous on bounded sets and hence (33) is shown to generate a local semiflow on $D\left(A_{p_{0}}^{\alpha_{0}}\right)$.

In order to derive a suitable a priori estimate for (33) let us multiply the first equation by $v$, integrate it over $(0, \pi)$ under the homogeneous boundary conditions, multiply the second equation by $U$ and next add the results. We obtain:

$$
\frac{1}{2} \frac{d}{d t}\left(\int_{0}^{\pi} v^{2} d x+U^{2}\right)=-\nu\left(\int_{0}^{\pi} v_{x}^{2} d x+U^{2}\right)+P U
$$

and further, using the Poincaré and Cauchy inequalities,

$$
\frac{1}{2} \frac{d}{d t}\left(\int_{0}^{\pi} v^{2} d x+U^{2}\right) \leq-\nu\left(\int_{0}^{\pi} v^{2} d x+U^{2}\right)+\frac{P^{2}}{2 \varepsilon}+\frac{\varepsilon U^{2}}{2}
$$

When $\varepsilon=\nu$ is inserted in (35), we come to the differential inequality

$$
\frac{d}{d t}\left(\int_{0}^{\pi} v^{2} d x+U^{2}\right) \leq-\nu\left(\int_{0}^{\pi} v^{2} d x+U^{2}\right)+\frac{P^{2}}{\nu}
$$


which leads to the estimate

$$
\int_{0}^{\pi} v^{2} d x+U^{2} \leq\left(\int_{0}^{\pi} v_{0}^{2} d x+U_{0}^{2}\right) e^{-\nu t}+\frac{P^{2}}{\nu} \frac{1-e^{-\nu t}}{\nu}
$$

so that asymptotically we get:

$$
\limsup _{t \rightarrow+\infty}\left(\int_{0}^{\pi} v^{2} d x+U^{2}\right) \leq \frac{P^{2}}{\nu^{2}} \text { for all }\left(v_{0}, U_{0}\right) \in D\left(A_{p_{0}}^{\alpha_{0}}\right) .
$$

Note that by [TR, Th. 5.5.1] the resolvent of $A_{1}$ is compact. Obtaining (11) is now purely technical and the existence of a global attractor for (33) follows (see Theorem 4 of Section 4). More precisely, estimating the leading nonlinear term of $F_{p}$ with the use of the Young inequality:

$$
\left|\frac{1}{2}\left(v^{2}\right)_{x}\right|=\left|v v_{x}\right| \leq \frac{3}{4}\left|v_{x}\right|^{\frac{4}{3}}+\frac{1}{4}|v|^{4},
$$

we may achieve a global attractor on the whole space $D\left(A_{p_{0}}^{\alpha_{0}}\right)$ with parameters $\alpha_{0}, p_{0}$ satisfying $2 \alpha_{0}-\frac{1}{p_{0}}>1$ and the exponent $\alpha_{0} \in\left(\frac{3}{4}, 1\right)$ (cf. (42) with $\left.\gamma_{0}=4, \gamma_{1}=\frac{4}{3}\right)$.

\subsection{Pattern formation equations}

The final group of examples for the study of which our theoretical approach of Section 2.1 was in fact created is formed by the pattern formation equations, namely the Cahn-Hilliard and Kuramoto-Sivashinsky equations. There is no need to repeat here detailed studies of these problems and its generalization - the Cahn-Hilliard system - since they were presented chronologically in [CD1]-[CD3] and earlier papers cited there.

\section{Technicalities}

\subsection{Problems with uniformly strongly parabolic operators}

Due to the complicated character of the definition of the regular parabolic initial boundary value problem to which we refer in the Assumption A-I, direct verification of its conditions is, in many examples, a rather uneasy operation. Slightly more convenient in applications is the situation when $\mathcal{A}$ is a uniformly strongly parabolic operator since, as may be seen from the results of [AM, Chapt. II], the Assumption A-I is then much simpler to prove.

Let the following smoothness conditions be satisfied: 
- All components of matrices $\left[a_{\alpha}^{r s}(x)\right]_{d \times d}(|\alpha| \leq 2 m)$ appearing in the definition of $\mathcal{A}$ belong to $C^{0}(c l \Omega)$.

- All elements of matrices $\left[b_{\beta}^{r s}(x)\right]_{1 \times d}\left(j=1, \ldots, m d,|\beta| \leq m_{j}\right)$, connected with boundary operators $\mathcal{B}_{j}$, are of the class $C^{2 m-m_{j}}(\partial \Omega)$.

Citing then [AM, Chapt. II, Sec. 6] (cf. also [LSU, Chapt. VII, §8]) we have:

Definition 4 A differential operator $\mathcal{A}:=(-1)^{m} \sum_{|\alpha| \leq 2 m}\left[a_{\alpha}^{r s}(x)\right]_{d \times d} D^{\alpha}$ is uniformly strongly parabolic iff

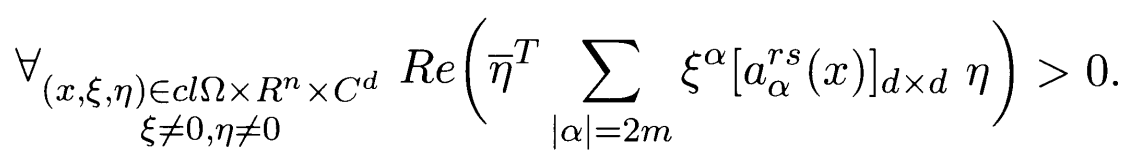

The result stated below is due to [AM, Chapt. II, Th. 6.6] and contains the general and simple criteria for validity of Assumption A-I for a large number of parabolic problems.

Proposition 4 Suppose that smoothness conditions hold and consider requirements (i), (ii) introduced below.

(i) $\mathcal{A}$ is a second order operator $(m=1)$, so that $\mathcal{A}$ admits the form

$$
\mathcal{A}=-\sum_{j, k=1}^{d}\left[a_{j k}^{r s}\right]_{d \times d} \frac{\partial^{2}}{\partial x_{j} \partial x_{k}}+\sum_{j}^{d}\left[a_{j}^{r s}\right]_{d \times d} \frac{\partial}{\partial x_{j}}+\left[a_{0}^{r s}\right]_{d \times d}
$$

and for some diagonal matrix $D=\left[\delta^{r s}\right]_{d \times d}$, where $\left(\delta^{11}, \ldots, \delta^{d d}\right) \in\{0,1\}^{d}$ :

$$
\begin{aligned}
& {[\mathcal{B}]_{d \times d}=D \sum_{j, k=1}^{d}\left[a^{r s}(x)_{j k}\right]_{d \times d} } \cos \left(N(x), x_{j}\right) \frac{\partial}{\partial x_{k}} \\
&+(I d-D)+D\left[b_{0}^{r s}(x)\right]_{d \times d}
\end{aligned}
$$

(ii) Vector fields $\nu_{i}(x) \in C^{2 m-1}\left(\partial \Omega, R^{n}\right)(i=1, \ldots, m)$ are defined, for which

$$
\exists_{c_{3}>0} \forall_{x \in \partial \Omega} \forall_{i=1, \ldots m} \nu_{i}(x) N(x) \geq c_{3}
$$

(where $N(x)$ denotes a normal vector to $\partial \Omega$ in $x \in \partial \Omega$ ) and there is $l \in$ $\{0, \ldots, m\}$ such that for $j=1, \ldots, m$ : 


$$
\left[\mathcal{B}_{j}\right]_{d \times d}=\left[\begin{array}{ccc}
\frac{\partial^{l+j-1}}{\partial \nu_{j}^{l+j-1}} & & 0 \\
0 & \ddots & \frac{\partial^{l+j-1}}{\partial \nu_{j}^{l+j-1}}
\end{array}\right]_{d \times d} \begin{array}{r}
+ \text { terms of order lower } \\
\text { than }(l+m-1) .
\end{array}
$$

If either the operator $\mathcal{A}$ is uniformly strongly parabolic and (ii) holds or $\mathcal{A}$ is uniformly very strongly parabolic and (i) is satisfied (cf. [AM1]), then the triple $\left(\mathcal{A},\left\{\mathcal{B}_{j}\right\}, \Omega\right)$ forms a regular parabolic initial boundary value problem, as is required in the Assumption A-I.

It is clear that Prop. 4 is not applicable to all problems of the type (1) for which Assumption A-I holds. For the remaining class of problems appearing in applications, either all conditions required in A-I must be separately verified or the sectorial property of $\mathcal{A}$, crucial for considerations of Sec. 2.1 needs to be justified, depending which is actually less difficult to show.

Below, the simple conditions allowing the abstract requirements of theorems of Sec. 2.1 to be realized will be studied.

\subsection{Realization of abstract conditions of Section 2.1.}

As a result of abstract considerations of (1) the existence of sufficiently smooth local solutions of (1) is guaranteed (see Prop. 2) so that the estimation procedure, in which both differential and integral properties of the solution are usually applied, may be continued until the required $\left[W^{l, r}(\Omega)\right]^{d_{-}}$ estimate of solutions is obtained. From the point of view of practical calculations it is unpleasant to be forced to verify abstract criteria; hence, in the following theorem the conditions of Section 2.1 have been formulated either in algebraic form or as $\left[W^{l, r}(\Omega)\right]^{d}$-type estimates which may be obtained from (1) with the use of standard techniques.

Theorem 4 Let $p_{0}, \alpha_{0}$ satisfy (4) and $V \subset D\left(A_{p_{0}}^{\alpha_{0}}\right)$. Then the conjunction of conditions (13) and (16) is equivalent to Conditions $\mathrm{H}_{1}-\mathrm{H}_{3}$ stated below.

Conditions $\mathrm{H}_{1}-\mathrm{H}_{3}$. There are some $l \geq 0, r \geq 1$ satisfying

$$
l<2 m \alpha_{0}, \quad r \leq p_{0}
$$

such that, as long as $D\left(A_{p_{0}}^{\alpha_{0}}\right)$-solutions $u\left(t, u_{0}\right)$ corresponding to $u_{0} \in V$ 
exist, the following properties hold:

$\left(\mathrm{H}_{1}\right) \quad$ For solutions starting from $u_{0} \in V a\left[W^{l, r}(\Omega)\right]^{d}$ estimate of the 'net' smoothness $s=l-\frac{n}{r}$ is known, i.e.

$$
\begin{aligned}
& \forall_{u_{0} \in V} \exists_{C_{1}\left(u_{0}\right)>0}\left\|u\left(t, u_{0}\right)\right\|_{\left[W^{l, r}(\Omega)\right]^{d}} \leq C_{1}\left(u_{0}\right), \text { where } \\
& C_{1}: V \rightarrow[0, \infty) \text { is bounded in bounded subsets of } V .
\end{aligned}
$$

$\left(\mathrm{H}_{2}\right) \quad$ On solutions originated in $u_{0} \in V$ the growth of the nonlinear term $f$ is limited according to a condition

$$
\begin{aligned}
& \left|f\left(x, u\left(t, u_{0}\right), D^{1} u\left(t, u_{0}\right), \ldots, D^{k} u\left(t, u_{0}\right)\right)\right| \\
& \quad \leq g\left(\left\|u\left(t, u_{0}\right)\right\|_{\left[W^{l, r}(\Omega)\right]^{d}}\right)\left(1+\sum_{j=0}^{k}\left|D^{j} u\left(t, u_{0}\right)\right|^{\gamma_{j}}\right)
\end{aligned}
$$

where $g:[0, \infty) \rightarrow[0, \infty)$ is nondecreasing and

$$
\begin{aligned}
& 1 \leq \gamma_{j}<1+\frac{2 m \alpha_{0}-j}{j-s} \quad \text { if } s<j, \\
& 1 \leq \gamma_{j} \text { arbitrarily large if } s \geq j .
\end{aligned}
$$

$\left(\mathrm{H}_{3}\right)$ The estimate $(40)$ is asymptotically independent of $u_{0} \in V$, i.e.

$$
\exists_{C_{2}>0} \forall_{u_{0} \in V} \limsup _{t \rightarrow+\infty}\left\|u\left(t, u_{0}\right)\right\|_{\left[W^{l, r}(\Omega)\right]^{d}} \leq C_{2} .
$$

Proof. If (13) and (16) hold, then using equivalent conditions of Prop. 3 and Th. 2 we obtain all requirements $\mathrm{H}_{1}-\mathrm{H}_{3}$. To prove the converse, note that thanks to (42) and our general assumption (4) there is some $\theta \in(0,1)$ such that

$$
1 \leq \gamma_{j}<\theta+\frac{\theta\left(2 m \alpha_{0}-j\right)+(1-\theta) \frac{n}{p_{0}}}{j-s}
$$

whenever $s<j$. Hence, the generalized Nirenberg-Gagliardo interpolation inequality of [AM, Cor. 4.2] can be applied and (13) follows after similar calculations as in $\left[\mathrm{CD} 2\right.$, Lem. 1] with $Y:=\left[W^{l, r}(\Omega)\right]^{d}$. Since $\mathrm{H}_{3}$ is then the same as (16), the proof is completed.

Remark 6. It is clear that $u\left(t, u_{0}\right)$ does not 'blow-up' for $u_{0} \in V$ if, and only if, Conditions $\mathrm{H}_{1}-\mathrm{H}_{2}$ hold. Furthermore, since $p_{0}$ can be taken arbitrarily large and $\alpha_{0}$ may be sufficiently close to 1 then both (4) and (39) are not 
restrictive at all whereas (42), roughly speaking, may be given in the form:

$$
\begin{aligned}
& 1 \leq \gamma_{j}<1+\frac{2 m-j}{j-s} \text { if } s<j \\
& 1 \leq \gamma_{j} \text { arbitrarily large if } s \geq j
\end{aligned}
$$

where parameter $s=l-\frac{n}{r}$ measures the 'net' smoothness of the estimate (40). Whenever $f$ is bounded by a power function, i.e. using symbolic notation when:

$$
\left|f\left(x, D^{0}, D^{1}, \ldots, D^{k}\right)\right| \leq \operatorname{const}\left(1+\sum_{j=0}^{k}\left|D^{j}\right|^{\gamma_{j}}\right)
$$

it is easy to calculate from (44) the minimal 'net' smoothness $s$ of the a priori $\left[W^{l, r}(\Omega)\right]^{d}$ estimate (40) necessary for validity of Conditions $\mathrm{H}_{1}-\mathrm{H}_{3}$. For example, if $m=1$ and $f\left(x, D^{0}, D^{1}\right)=\left|D^{0}\right|^{\text {any number }}+\left|D^{1}\right|^{2}$ then it is necessary to derive the estimate whose 'net' smoothness $s$ is positive (i.e. an estimate slightly 'better' then $L^{\infty}$ must be known); whereas for $m=1$ and $f\left(x, D^{0}, D^{1}\right)=\left|D^{0}\right|^{\text {any number }}+\left|D^{1}\right|^{2-\varepsilon}(\varepsilon \in(0,1))$ it suffices to have the estimate with 'net' smoothness $s>\frac{-\varepsilon}{1-\varepsilon}$ (i.e. $L^{\infty}$ estimate is then sufficient).

As a consequence of Th. 3 and Th. 4 we obtain:

Corollary 3 Let $p_{0}, \alpha_{0}$ satisfy $(4),\{T(t)\}$ be a local semiflow on $D\left(A_{p_{0}}^{\alpha_{0}}\right)$ resulting from Prop. 2 and the resolvent of $A_{p_{0}}$ be compact. Then $\{T(t)\}$ has a restricted global attractor in $D\left(A_{p_{0}}^{\alpha_{0}}\right)$ if, and only if, Conditions $\mathrm{H}_{1}-$ $\mathrm{H}_{3}$ hold with some closed and positively invariant nonempty subset $V$ of $D\left(A_{p_{0}}^{\alpha_{0}}\right)$.

Although, from a theoretical point of view, the existence of restricted global attractors for parabolic problems of type (1) has been completely settled here, it is always a delicate and crucial point in applications to find sufficiently 'net'-smooth a priori estimate of solutions. However, no general method to realize this point may be developed and results above merely answer the question of which is the minimal 'net' smoothness of the a priori estimate that should be known.

Acknowledgment The first author would like to acknowledge the support from NSF and the hospitality of the University of Nebraska at Lincoln, where part of this paper was carried out. The authors are also grateful to the Referee for a number of comments improving the redaction of the paper. 


\section{References}

[AD] Adams R.A., Sobolev Spaces. Academic Press, New York, 1975.

[AM] Amann H., Global existence for semilinear parabolic systems. J. Reine Angew. Math. 360 (1985), 47-83.

[AM1] Amann H., Parabolic evolution equations and nonlinear boundary conditions. J. Differential Equations 72 (1988), 201-269.

[C] Carvalho A.N. de, Contracting sets and dissipation. Proc. Roy. Soc. Edinburgh 125A (1995), 1305-1329.

[CRF] Carvalho A.N. de and Ruas-Filho J. G., Global attractors for parabolic problems in fractional power spaces. SIAM J. Math. Analysis 26 (2) (1995), 415-427.

[CD1] Cholewa J.W. and Dlotko T., Global attractor for the Cahn-Hilliard system. Bull. Austral. Math. Soc. 49 (1994), 277-293.

[CD2] Cholewa J.W. and Dlotko T., Global solutions via partial information and the Cahn-Hilliard equation. in: Symposium on Singularities and Differential Equations, 1993, Banach Center Publications (PWN), Warsaw, 1996, pp. 39-50.

[CD3] Cholewa J.W. and Dlotko T., Global attractor for sectorial evolutionary equation. J. Differential Equations 125 (1996), 27-39.

[CCS] Chueh K.N., Conley C.C. and Smoller J.A., Positively invariant regions for systems of nonlinear diffusion equations. Indiana Univ. Math. J. 26 (1977), 373-392.

[DL] Dlotko T., The two-dimensional Burgers' turbulence model. J. Math. Kyoto Univ. 21, 4 (1981), 809-823.

[FR] Friedman A., Partial Differential Equations. Holt, Rinehart and Winston, New York, 1969.

[GM] Giga Y. and Miyakawa T., Solutions in $L_{r}$ of the Navier-Stokes initial value problem. Arch. Rational Mech. Anal. 89 (3) (1985), 267-281.

[HA] Hale J.K., Asymptotic Behavior of Dissipative Systems. AMS, Providence, R. I., 1988.

[HE] Henry D., Geometric Theory of Semilinear Parabolic Equations. Springer-Verlag, Berlin, 1981.

[LSU] Ladyẑenskaja O.A., Solonnikov V. A. and Ural'ceva N.N., Linear and Quasilinear Equations of Parabolic Type. Nauka, Moscow 1967.

[PR] Promislow K., Time analyticity and Gevrey regularity for solutions of a class of dissipative partial differential equations. Nonlinear Analysis TMA 11 (16) (1991), 959-980.

[RO] Rothe F., Global Solutions of Reaction-Diffusion Systems. Springer-Verlag, Berlin, 1984.

[RS] Rauch G., Smoller J., Qualitative theory of the Fitz Hugh-Nagumo equations. Advances Math. 27 (1978), 12-44.

[SM] Smoller J., Shock Waves and Reaction-Diffusion Equations. Springer-Verlag, New York, 1988.

[TE] Temam R., Infinite - Dimensional Dynamical Systems in Mechanics and Physics. Springer-Verlag, New York, 1988. 
[TR] Triebel H., Interpolation Theory. Function Spaces, Differential Operators, Veb Deutscher Verlag, Berlin, 1978, also; North-Holland, Amsterdam, 1978.

Alexandre N. Carvalho

Departamento de Matemática do ICMSC

Universidade de São Paulo

13.560 São Carlos - S. P. - Brazil

E-mail: andcarva@icmsc.sc.usp.br

Jan W. Cholewa

Institute of Mathematics

Silesian University

40-007 Katowice, Poland

E-mail: jcholewa@gate.math.us.edu.pl

Tomasz Dlotko

Institute of Mathematics

Silesian University

40-007 Katowice, Poland

E-mail: tdlotko@gate.math.us.edu.pl 